Trauma Berufskrankh 2012 · 14[Suppl 3]:263-267 DOI 10.1007/s10039-012-1854-0

Online publiziert: 25. Mai 2012

(c) Springer-Verlag 2012

A. Kranig

Abteilung Versicherung und Leistungen, Deutsche Gesetzliche Unfallversicherung (DGUV), Berlin

\title{
Zukünftiges stationäres Heilverfahren
}

\section{Aus Sicht der DGUV (Deutsche Gesetzliche Unfallversicherung)}

\section{Neuausrichtung der stationären Heilverfahren}

Für diese Neuausrichtung gibt es verschiedene Gründe. Die Wichtigsten sind:

\section{Strukturveränderungen}

Die Versorgungsstrukturen im Krankenhausbereich haben sich verändert und entwickeln sich weiter. Viele Krankenhäuser organisieren sich neu, wodurch einige nicht mehr den Anforderungen, die heute an eine qualitativ hochstehende unfallchirurgische Versorgung rund um die Uhr zu stellen sind, genügen.

Die Deutsche Gesellschaft für Unfallchirurgie (DGU) setzte diesen erkennbaren Entwicklungen durch das Weißbuch [1] und die Bildung des TraumaNetzwerk $\mathrm{DGU}^{\circledR}$ eine Qualitätsoffensive entgegen: Hieran kann die Deutsche Gesetzliche Unfallversicherung (DGUV) anknüpfen, um dem Auftrag der Heilbehandlung und Rehabilitation mit allen geeigneten Mitteln gerecht zu werden.

\section{Änderung des Bedarfs}

Der Bedarf der gesetzlichen Unfallversicherung (GUV) ändert sich: Rückläufige Fallzahlen legen eine Konzentration auf die leistungsfähigsten und bestqualifizierten Krankenhäuser nahe, ebenso aber auch die Fokussierung auf die schweren und schwersten Verletzungen, die sowohl aus sozialen und humanitären als auch aus wirtschaftlichen Gründen geboten ist.

\section{Qualitätssicherung}

Wir sind sowohl der Politik, die unseren gesetzlichen Auftrag formulierte, als auch den Unternehmern und Versicherten als Träger der GUV zum Nachweis von Qualität und Effizienz unserer Aktivitäten verpflichtet - und dies wird zu Recht zunehmend eingefordert. Dabei haben wir die Perspektive der Betroffenen verstärkt zu beachten.

\section{Nutzung des TraumaNetzwerk DGU ${ }^{\circledR}$}

\section{Übertragbare Strukturen}

Mit dem TraumaNetzwerk DGU ${ }^{\circledast}$ entsteht derzeit eine an Qualität und Effizienz ausgerichtete, gestufte Versorgungsstruktur. Sie kann der Neuordnung unserer Heilverfahren als Blaupause dienen. An dem 3-stufigen Aufbau mit

- Häusern der Grund- und Regelversorgung als Basis,

- unfallchirurgisch hochqualifizierten Häusern als regionale Zentren und

- Maximalversorgern auf der obersten Versorgungsstufe

können wir auch unsere Strukturen ausrichten.

Die Zertifizierung aufgrund definierter Struktur- und Prozessanforderungen können wir als Grundqualifikationen auch für unsere Heilverfahren zugrunde legen und um unsere Spezifika ergänzen.
Wichtig ist insbesondere eine gleichgerichtete Abstimmung mit den Rettungsleitstellen: Die Zuweisung der Patienten nach Art und Schwere ihrer Verletzung wird im Sinn der Steuerung unserer Heilverfahren nur dann optimal funktionieren, wenn unsere Strukturen den allgemein für die Versorgung Unfallverletzter bestehenden weitgehend entsprechen.

\section{Aus Sicht der GUV notwendige Ergänzungen}

Das TraumaNetzwerk DGU ${ }^{\circledR}$ ist auf die Optimierung der Akutversorgung fokussiert. Die umfassende Sicht der GUV - entsprechend dem Grundsatz alles aus einer Hand - erfordert die stärkere Einbeziehung und Verzahnung mit der medizinischen und ggf. beruflichen Rehabilitation sowie - insbesondere auch in Komplikationsfällen - die Rekonstruktion. Dies betrifft die von Beginn an schweren und schwersten Verletzungen, aber auch die komplizierten Verläufe. Hierzu gehören die früheren Denkschriftfälle - insbesondere schwere Brandverletzungen, Querschnittslähmungen und SchädelHirn-Traumen, zudem Komplikationsfälle sowie Fälle, die wegen der Prognose langer Arbeitsunfähigkeit oder aus anderen Gründen durch das Rehabilitationsmanagement der UV-Träger betreut werden müssen.

Diese Fälle mit besonderer Relevanz für die GUV wollen wir in die oberste Stufe eines GUV- oder SGB-VII-Traumanetzwerks (SGB: Sozialgesetzbuch) steu- 


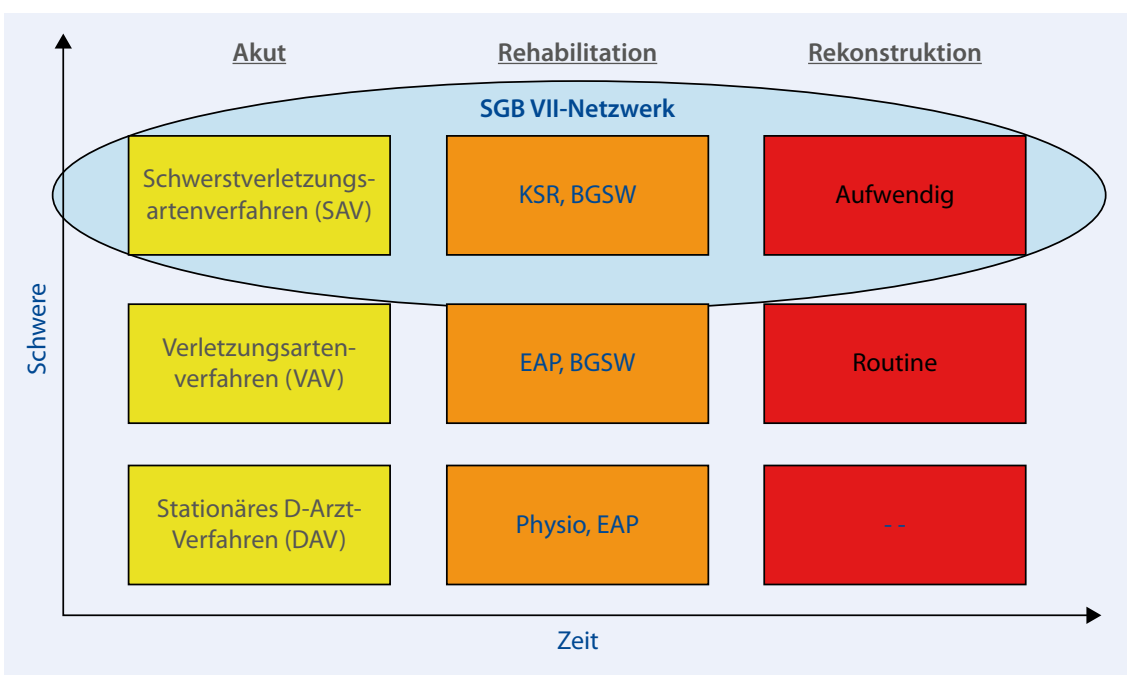

Abb. 1 \ Grundstruktur des Netzwerks zur stationären Versorgung in der gesetzlichen Unfallversicherung, $B G S W$ berufsgenossenschaftliche stationäre Weiterbehandlung, D-Arzt Durchgangsarzt, EAP erweiterte ambulante Physiotherapie, KSR komplexe stationäre Rehabilitation, SGB Sozialgesetzbuch

ern. Dort sind die 9 BG-Kliniken (BG: Berufsgenossenschaft) und weitere Maximalversorger in bedarfsgerechter Zahl angesiedelt, sie bilden den Kern dieses Netzwerks.

All dies muss für alle Beteiligten insbesondere die UV-Träger, die Kliniken und die Rettungsdienste - praktikabel und transparent ausgestaltet werden. Hierzu muss der Verletzungsartenkatalog so überarbeitet werden, dass sich schnell und übersichtlich ablesen lässt, welche Versorgungsstufe im Netzwerk anzusteuern ist.

\section{Ausgestaltung der GUV-Netzwerks zur stationären Versorgung}

In den bisherigen Diskussionen innerhalb der GUV, aber auch mit der Ärzteschaft und den Kliniken bildete sich bereits ein sehr weitgehender Konsens über die Grundstruktur des Netzwerks zur stationären Versorgung in der GUV heraus (• Abb. 1). Die künftigen 3 Versorgungsstufen im Netzwerk sind wie folgt zu charakterisieren.

\section{Basisversorgung}

Auf der ersten Stufe erfolgt die Grundund Regelversorgung aller Akutfälle, die nicht durch den überarbeiteten Verlet- zungsartenkatalog der 2. oder 3. Stufe vorbehalten sind.

Die rehabilitative Versorgung auf dieser Stufe erfolgt durch ambulante Physiotherapie, bei Bedarf in Form der erweiterten ambulanten Physiotherapie (EAP).

Im Zuge der Überarbeitung des Verletzungsartenkatalogs werden in Zukunft weniger Verletzungsarten der 2. und 3. Stufe vorbehalten bleiben - der Anteil, der auf der ersten Stufe zu behandelnden Verletzungen soll also einerseits steigen, andererseits aber auf die Fälle beschränkt sein, denen bereits diese Versorgungsstufe voll gerecht wird.

Bislang sind die Rechtsbeziehungen auf dieser Stufe beschränkt: Sie bestehen lediglich zwischen der DGUV und den stationär tätigen Ärzten. In Zukunft sollen sie auf die formell zum DAV (Durchgangsarztverfahren) zugelassenen Krankenhäuser erweitert werden. Die Häuser, die künftig nicht mehr am VAV teilnehmen können, werden damit - sofern sie weiterhin in relevanter Weise Unfallchirurgie betreiben - ausgewiesene Partner der GUV bleiben. Damit wird auf der Stufe der Grund- und Regelversorgung ein dichtes Netz sichergestellt - wesentlich beispielsweise für Versichertengruppen, die unabhängig von industriellen Ballungsbereichen übers ganze Land verteilt sind, wie Kinder, Schüler oder in der Landwirtschaft Tätige.

\section{Zweite Versorgungsstufe}

Auf dieser Stufe - dem modifizierten Verletzungsartenverfahren (VAV) - streben wir eine Konzentration im Sinn von Qualitäts- und Effizienzsteigerung an.

Wie bereits angeführt, ist unser quantitativer Bedarf gesunken, der qualitative dagegen steigt. Ersterer liegt deutlich unter den derzeit etwa 600 zugelassenen VAV-Häusern. Hier zeigt sich eine Parallele zum TraumaNetzwerk DGUं, denn auch dort zeichnet sich eine Größenordnung von etwa 300 Häusern oberhalb der Grund- und Regelversorgung ab. Häuser, die dort auf der 2. Stufe der regionalen Traumazentren angesiedelt sind, werden regelhaft auch die Voraussetzungen des VAV erfüllen. Mit der Zertifizierung zum TraumaNetzwerk DGU ${ }^{\circ}$ werden wesentliche Qualitätsanforderungen erfüllt, die auch für den Bereich der GUV gelten. In diesem Zusammenhang sei daran erinnert, dass das Weißbuch [1] und das TraumaNetzwerk DGU ${ }^{\circ}$ an die guten Erfahrungen mit dem bisherigen VAV der GUV anknüpfen und dies - bezogen auf die gesamte unfallchirurgische Versorgung in Deutschland - verallgemeinern und fortentwickeln. Dass sich die DGUV hierauf bei der Neuordnung ihrer Heilverfahren wiederum bezieht, ist nur folgerichtig.

Als Indikator für eine optimale unfallchirurgische Versorgung ist vorgesehen, die Zulassung zum VAV grundsätzlich von einer Mindestfallzahl von im VAV behandelten Unfallverletzten abhängig zu machen. Sie soll ausreichende Übung bei der Behandlung Schwerverletzter ebenso gewährleisten wie Vertrautheit mit den besonderen Verfahrens- und Qualitätsanforderungen der GUV. Nach dem jetzigen Stand wird die Mindestfallzahl über 50 und unter 100 liegen. Mit einem Wert in der Mitte dieses Bereichs würde erreicht, dass pro Kalendertag etwa eine Verletzung zu behandeln wäre, deren Schwere dem Verletzungsartenkatalog der GUV entspricht. (Die GUV ist bekanntlich für etwa $1 / 5$ der in Deutschland eintretenden Unfälle zuständig.) Dies dürfte für eine ausreichende Übungshaltung ausreichen. Soweit die Anwendung der Mindestfallzahl zu Versorgungslücken in der Fläche führen würde, sieht das Konzept regionale Ausnahmen entsprechend dem tat- 
sächlichen Bedarf vor. Dieser richtet sich nicht nur nach der Zahl der Versicherten in der Region, sondern auch nach der verkehrsmäßigen Erreichbarkeit (entsprechend einem noch festzulegenden zeitlichen Höchstwert für den Transport vom Unfallort zum Krankenhaus).

VAV-Häuser sind natürlich nicht nur für die Verletzungen des Verletzungsartenkatalogs, sondern auch für die Grundund Regelversorgung zuständig. Hinsichtlich der Katalogverletzungen mündet die Akutversorgung regelhaft in medizinische Rehabilitationsmaßnahmen der EAP oder, soweit erforderlich, der berufsgenossenschaftlichen stationären Weiterbehandlung (BGSW). Auf der Ebene des VAV sollen auch routinemäßige Rekonstruktionsbehandlungen erfolgen, die nicht die besonderen Kapazitäten eines Maximalversorgers erfordern. Die Abgrenzung soll durch die neue Nr. 10 des Verletzungsartenkatalogs erfolgen.

\section{Kostenerstattung}

Die Vergütung soll wie bisher über die allgemeinen Fallpauschalen erfolgen. Soweit die GUV zusätzliche Leistungen beauftragt, z. B. ärztliche Mitwirkung an der Rehabilitationsplanung im Rahmen des Rehabilitationsmanagements oder Erstattung von Arztberichten, werden diese wie bisher gesondert vergütet. Durch die $\mathrm{Zu}$ steuerung schwerer Fälle kann sich der CMI-Wert (CMI: Case-Mix-Index) eines Hauses erhöhen, was sich bei der Bemessung der Fallpauschalen positiv auswirkt.

\section{Maximalversorgung}

Neu ist die ausdrückliche Ausweisung einer 3. Versorgungsstufe - des Schwerstverletzungsartenverfahrens (SAV). Sie soll bestimmten, im neuen Verletzungsartenkatalog definierten und grafisch herausgehobenen schwersten Verletzungen vorbehalten bleiben. Hierzu gehören die Behandlung von

- schweren Brandverletzungen,

- Querschnittslähmungen,

- Schädel-Hirn-Traumen,

- Trümmerbrüchen der großen Gelenke,

- schweren Fußverletzungen sowie

- aufwendige Rekonstruktionsbehandlungen.

Trauma Berufskrankh 2012 - 14[Suppl 3]:263-267 DOI 10.1007/s10039-012-1854-0

(c) Springer-Verlag 2012

\section{A. Kranig \\ Zukünftiges stationäres Heilverfahren. Aus Sicht der DGUV (Deutsche Gesetzliche Unfallversicherung)}

\section{Zusammenfassung}

Die Neuregelung der stationären Heilverfahren im Akutbereich soll nach derzeitigem Stand (Februar 2012) im Herbst 2012 beschlossen werden und zum 01.01.2013 in Kraft treten. In diesem Zusammenhang stellen sich folgende Fragen, die im vorliegenden Beitrag behandelt werden: Warum richtet die gesetzliche Unfallversicherung (GUV) ihre stationären Heilverfahren neu aus? Wie kann die GUV das TraumaNetzwerk DGU ${ }^{\circledR}$ (DGU: Deutsche Gesellschaft für Unfallchirurgie) nutzen und um welche Aspekte ist dieses aus Sicht der GUV zu ergänzen? Wie soll das Netzwerk zur stationären Versorgung in der GUV ausgestaltet werden? Welche Qualitätsmaßnahmen sind für das SAV (Schwerstverletzungsartenverfahren) einschließlich der
BG-Kliniken (BG: Berufsgenossenschaft) vorgesehen? Was ist im Jahr 2012 zu erwarten? Alle vorgesehenen Änderungen werden intensiv im Kreis der GUV und ihrer Selbstverwaltung, mit den Organisationen der Ärzteschaft und der Krankenhäuser erörtert und abgestimmt. Gemeinsam setzen GUV, DGU und die Berufsverbände der für die Unfallversicherungsträger tätigen Ärzte die richtigen Schwerpunkte im Interesse der Arbeitsunfallverletzten.

\section{Schlüsselwörter}

Unfallversicherung · Arbeitsunfälle .

Heilverfahren · Neuordnung der stationären Heilverfahren · Netzwerkbildung

\section{Future inhospital treatment. From the perspective of the DGUV (German statutory accident insurance)}

\section{Abstract}

According to the current state of affairs (February 2012) the new regulations for inhospital treatment in emergency situations should be completed in autumn 2012 and come into effect on $1^{\text {st }}$ January 2013. In connection with this the following questions arise which will be dealt with in this article: why is the statutory accident insurance (GUV) amending the inhospital treatment procedure? How can the GUV use the TraumaNetwork DGU ${ }^{\otimes}$ (DGU German Society for Trauma Surgery) and under which aspects can this be supplemented from the perspective of the GUV? How should the network be developed for inhospital treatment in the GUV? Which quality measures are intended for the SAV (severe in- jury type procedure) including the BG clinics (occupational insurance association)? What is to be expected in 2013? All intended amendments will be intensively discussed and ratified within the GUC and its administrative bodies, with the organisations of the medical profession and hospitals. The GUV, DGU and professional associations will determine the correct focal points for physicians actively involved with accident insurers in the interests of victims of occupational accidents.

\section{Keywords}

Accident insurance - Occupational accidents . Treatment $\cdot$ Amendments to inhospital treatment - Network formation
Für diese schwersten Verletzungen ist es zunächst erforderlich, alle geeigneten Mittel einer optimalen und häufig speziellen, interdisziplinär aufgestellten unfallchirurgischen Akutversorgung einzusetzen.

Darüber hinaus setzen die gesundheitliche Wiederherstellung und auch die berufliche, familiäre und gesellschaftliche Wiedereingliederung dieser Verletzten voraus, dass die Krankenhäuser auf dieser Stufe vielfältige Behandlungsmöglichkeiten vorhalten, die im Bedarfsfall abgerufen werden können. Diese Möglich- keiten an mehreren hundert Häusern in Deutschland bereitzuhalten, wäre weder bedarfsgerecht noch ökonomisch sinnvoll. Daher erfolgt auch hier eine an Bedarf und Qualität ausgerichtete Konzentration - wiederum in Übereinstimmung mit dem TraumaNetzwerk DGU ${ }^{\circledast}$ : Dort wird es im Endausbau voraussichtlich weniger als 100 überregionale Traumazentren geben. Auch hieran werden wir uns bei der Zulassung zum SAV grundsätzlich orientieren können. Für die Belange der GUV und ihrer sehr schwer Verletz- 


\section{$\sum$ Akut $\gg$ Frühreha $\gg B G S W \gg A B M R \gg$ Nachsorge $\gg$ Pflege}

Abb. $2 \Delta$ SGB-VII-Rehabilitationspfade für schwere Verletzungsfälle, $A B M R$ arbeitsplatzbezogene muskuloskelettale Rehabilitation, $B G S W$ berufsgenossenschaftliche stationäre Weiterbehandlung, Frühreha Frührehabilitation, $S G B$ Sozialgesetzbuch

ten sind u. a. folgende Behandlungsoptionen zentral wichtig:

- eine schon in der Akutphase so früh wie möglich einsetzende Frührehabilitation, die diesen Namen auch verdient,

- eine psychotraumatologische Diagnostik und Betreuung,

- die enge Kooperation mit den UVTrägern bei der Steuerung der Heilverfahren,

- eine Vernetzung und die Organisation unmittelbarer Anschlüsse mit den Einrichtungen der weiteren Rehabilitation [in der Regel BGSW, bei Bedarf arbeitplatzbezogene muskuloskelettale Rehabilitation (ABMR) oder komplexe stationäre Rehabilitation (KSR)].

Auf dieser Stufe waren bereits bislang die 9 BG-Unfallkliniken und weitere Kooperationshäuser tätig. Die BG-Kliniken werden auch im neuen Netzwerk eine herausragende Stellung im Rahmen der GUVHeilverfahren innehaben. Als Schrittmacher der Heilverfahren sind sie seit Anfang 2012 organisatorisch gemeinsam im Klinikverbund der gesetzlichen Unfallversicherung - KUV - neu aufgestellt. Sie sollen noch mehr als bisher - neben einer unfallchirurgischen Maximalversorgung einschließlich BGSW, ABMR und KSR alles unter einem Dach - spezielle Dienstleistungen für die GUV nach deren Bedarf erbringen. Beispiele sind die unterschiedlichen besonderen Sprechstunden zur Vorstellung bei problematischen Heilverläufen, Beratungsangebote für Ärzte in Kliniken der ersten und zweiten Versorgungsstufe, Fortbildungsangebote und Qualitätszirkel oder Pflegeberatung. Auch die Forschung zur Weiterentwicklung der medizinischen Behandlung Unfallverletzter wie zur Verbesserung, Erprobung und Validierung neuer Verfahren zählt hierzu.

Die BG-Kliniken können den Bedarf auf der 3. Stufe aber nicht allein abdecken.
Deswegen ist die Zulassung weiterer Häuser zum SAV vorgesehen, die sich aus den überregionalen Zentren des DGUV-Traumanetzwerks rekrutieren werden. Neben der dort zertifizierten besonderen unfallchirurgischen Kompetenz und Kapazität setzt die Zulassung zum SAV insbesondere voraus, dass Vorkehrungen für einen nahtlosen Anschluss erforderlicher Rehabilitationsmaßnahmen getroffen sind und die für diese Stufe vorgesehenen Standards eingehalten werden.

Die SAV-Häuser sind natürlich nicht auf die Behandlung Schwerstverletzter beschränkt, sondern auch für die Grundund Regelversorgung und das VAV zuständig. Hinsichtlich der im Verletzungsartenkatalog herausgehobenen schwersten SAV-Verletzungsarten mündet die Akutversorgung, wie oben erwähnt, regelhaft in die besonders qualifizierten Rehabilitationsmaßnahmen der BGSW, ABMR oder KSR. Die SAV-Häuser sollen insbesondere auch die aufwendigen Rekonstruktionen vornehmen. Als höchste Versorgungsstufe haben sie damit im Bereich der Akutversorgung Unfallverletzter eine Allzuständigkeit.

Die SAV-Häuser sollen ebenso wie alle anderen an den Heilverfahren der GUV beteiligten Ärzte und Krankenhäuser von den DGUV-Landesverbänden zugelassen werden. Eine enge Vernetzung mit den BG-Kliniken und die Teilnahme an gemeinsamer Qualitätssicherung werden zwar vorausgesetzt; neben der Zulassung durch die DGUV wird es hierfür aber keiner Sonderrechtsbeziehungen zu einer der BG-Kliniken oder dem KUV bedürfen.

\section{Kostenerstattung}

Der aktuelle Beratungsstand zur Vergütung der Leistungen der zum SAV zugelassenen Häuser stellt sich wie folgt dar: Sie soll sich generell für alle Verletzungsarten nach den allgemein geltenden Fallpauschalen richten, soweit solche gesetzlich aufgrund des Krankenhausentgeltge- setzes (KHEntgG) festgelegt sind. Soweit dies nicht der Fall ist, sind gesonderte (tagesgleiche) Pflegesätze zu vereinbaren. Wie schon oben zum VAV ausgeführt, wird die Steuerung schwerster Verletzungsfälle in das SAV den CMI der SAVHäuser steigern und sich somit leistungsgerecht erhöhend auf die Fallpauschalen auswirken. Soweit die GUV besondere, mit den Fallpauschalen nicht abgegoltene ärztliche Leistungen beauftragt (Berichte, Mitwirkung an der Rehabilitationsplanung), werden diese wie bisher gesondert vergütet.

\section{Für das SAV einschließlich der BG-Kliniken vorgesehene Qualitätsmaßnahmen}

Für die BG-Kliniken und die SAV-Häuser sollen folgende Standards erarbeitet, abgestimmt und verbindlich gemacht werden:

\section{Standards für die Kooperation mit den UV-Trägern, insbesondere mit deren Re- habilitationsmanagement. Grundlage hierfür ist der von den UV-Trägern auf der Ebene der DGUV gemeinsam erarbei- tete und 2010 beschlossene Handlungs- leitfaden zum Rehabilitationsmanage- ment [2]; er wird derzeit durch konkre- te Handlungshilfen, z. B. ein Formtexteset zur Rehabilitationsplanung, unterfüttert. \\ Standards der Medizin und Therapie. Sie sollen insbesondere in Rehabilitationspfa- den für schwere Verletzungsarten nieder- gelegt und nach dem Vorbild der neurolo- gischen Rehabilitation als Phasenmodelle dargestellt werden (• Abb. 2).}

\section{Standards der Vernetzung mit ande- ren Leistungserbringern; insbesondere mit Rehabilitationskliniken und ande- ren Rehabilitationseinrichtungen. So- weit Rehabilitationsmaßnahmen nicht im eigenen Haus - ohne Schnittstellen - erbracht werden können, ist ein nahtloser Anschluss zwischen Akutbehandlung und Rehabilitation sicherzustellen.}

\section{Im Jahr 2012 zu erwartende Maßnahmen}

Die Neuregelung der stationären Heilverfahren im Akutbereich soll nach derzeitigem Stand (März 2012) im Herbst 2012 be- 
schlossen werden und zum 01.01.2013 in Kraft treten. Hierfür sind die erforderlichen Vorarbeiten weit gediehen, bedürfen aber noch des Feinschliffs und der weiteren Beratung, sowohl in den Gremien der DGUV und der UV-Träger als auch mit den Vertretungen der Ärzteschaft und Krankenhäuser. Die Landesverbände der DGUV werden die Neuregelung ab 2013 umsetzen; dabei werden die positiven $\mathrm{Zu}$ lassungen zum DAV, VAV und SAV sofort wirksam, während die Beendigung von Beteiligungen am VAV regelhaft erst nach einer Übergangsfrist wirksam wird - es sei denn, bisherige zum VAV zugelassene Kliniken erklären ihre Bereitschaft, mit sofortiger Wirkung für das stationäre DAV zu optieren. Einvernehmliche Regelungen dieser Art fördern den zügigen Übergang zu den neuen Strukturen. Sie können auch im Interesse der betroffenen Häuser liegen, insbesondere wenn einer geringen Zahl von Verletzungsartenkatalogverletzungen ein hoher Aufwand an erforderlichen Vorhaltekosten und Investitionen gegenübersteht. Die DGUV-Landesverbände werden das Gespräch mit Häusern suchen, die die neuen Voraussetzungen für eine VAV-Beteiligung voraussichtlich nicht erfüllen werden.

Bereits jetzt (Stand: Februar 2012) vorliegende Vorarbeiten sind:

Entwurf des überarbeiteten Verletzungsartenkatalogs. Er soll auch die Kataloge zum SAV und zu den der obersten Stufe vorbehaltenen Komplikationsarten enthalten (- Abb. 1). Die im Verletzungsartenkatalog definierten Verletzungsarten sollen den VAV- und den SAV-Häusern vorbehalten sein, die besonders hervorgehobenen Verletzungs- und Komplikationsarten ausschließlich den SAVHäusern. Alle nicht im Verletzungsartenkatalog aufgeführten Verletzungsarten können in Häusern aller 3 Stufen behandelt werden. Damit lässt sich grundsätzlich aus den 10 vorgesehenen Positionen des Verletzungsartenkatalogs ablesen, in welche Art von Krankenhäusern Verletzungsfälle zu steuern sind.

Entwurf der Erläuterungen zum Verletzungsartenkatalog. Zu den 10 angeführten, notwendigerweise abstrakt definierten Positionen besteht natürlich Erläute- rungsbedarf. Auch der Entwurf der Erläuterungen zum Verletzungsartenkatalog liegt vor.

Entwurf zu den Anforderungen an Krankenhäuser. Auch die Anforderungen zur Beteiligung von Krankenhäusern an der stationären Behandlung Arbeitsunfallverletzter müssen neu gefasst werden, wenn die dargestellten neuen Strukturen wirksam werden sollen. Und auch für diese Anforderungen liegt nunmehr ein Entwurf vor. Die erwähnten SAV-Standards der Kooperation mit den UV-Trägern und der Vernetzung mit anderen Leistungserbringern sind in diesen Entwurf eingearbeitet.

Rehabilitationspfade. Einige der angestrebten Standards der Medizin und Therapie (Rehabilitationspfade) sollen noch im Jahr 2012 erarbeitet werden (zu Schädel-Hirn-Verletzungen, zu Trümmerbrüchen der großen Gelenke und zu schweren Fußverletzungen). Nach dem Entwurf der oben angeführten Anforderungen sollen sie für die SAV-Häuser Schritt für Schritt verbindlich werden, sobald die einzelnen Rehabilitationspfade verabschiedet sind.

\section{Ausblick}

Alle vorgesehenen Änderungen werden intensiv - nicht nur im Kreis der GUV und ihrer Selbstverwaltung, sondern auch mit den Organisationen der Ärzteschaft und der Krankenhäuser - erörtert und abgestimmt. Insbesondere nimmt die Neuordnung der GUV-Heilverfahren Entwicklungen auf, die mit dem Weißbuch der DGU [1] und dem darauf fußenden Traumanetzwerk von der Unfallchirurgie selbst initiiert und bereits weithin umgesetzt wurden. Gemeinsam setzen die DGUV, die DGU und die Berufsverbände der für die UV-Träger tätigen Ärzte die richtigen Schwerpunkte im Interesse der Arbeitsunfallverletzten:

- bei der optimalen Akutversorgung schwerer und schwerster Verletzungen,

- bei einer frühen, nachhaltigen, arbeitsbezogenen medizinischen Rehabilitation und
- bei der Optimierung der Versorgung bei Komplikationen.

Dadurch werden die vorhandenen Ressourcen noch besser eingesetzt und vernetzt,

- insbesondere wird das VAV gestrafft, konzentriert und damit gestärkt, aber auch

- die flächendeckende Grund- und Regelversorgung im stationären DAV sichergestellt, bei Ausweitung der dort zu behandelnden Verletzungsarten durch Abspecken des Verletzungsartenkatalogs.

Unfallversicherung und Unfallchirurgie erweisen sich im Prozess der Neuordnung der GUV-Heilverfahren als gute und verlässliche Partner - sie werden dies auch in den zukünftigen erneuerten Strukturen auf allen Ebenen bleiben.

\section{Korrespondenzadresse}

\section{Dr. A. Kranig}

Abteilung Versicherung und Leistungen,

Deutsche Gesetzliche Unfallversicherung (DGUV),

Mittelstraße 51, 10117 Berlin

Andreas.kranig@dguv.de

Interessenkonflikt. Der korrespondierende Autor gibt an, dass kein Interessenkonflikt besteht.

The supplement containing this article is not sponsored by industry.

\section{Literatur}

1. Deutsche Gesellschaft für Unfallchirurgie (DGU) (2006) Weißbuch Schwerverletzten-Versorgung Empfehlungen zur Struktur, Organisation und Ausstattung der Schwerverletzten-Versorgung in der Bundesrepublik Deutschland. DGU, Berlin

2. Deutsche Gesetzliche Unfallversicherung (DGUV) (2010) Das Reha-Management der Deutschen Gesetzlichen Unfallversicherung - Handlungsleitfaden. DGUV, Berlin 